\title{
La justicia espacial y su aplicabilidad en espacios públicos de México
}

\author{
Spatial justice and its applicability in Mexican public spaces
}

Elizabeth Sedano

Tecnológico Nacional de México. Instituto Tecnológico de Colima (México)

Peter Chung Alonso

Tecnológico Nacional de México. Instituto Tecnológico de Colima (México)

María Silvia del Rocío Covarrubias Ruesga

Tecnológico Nacional de México. Instituto Tecnológico de Colima (México)

Sedano, E., Chung Alonso, P., \& Covarrubias Ruesga, M. S. del R. (202I). La Justicia Espacial y su aplicabilidad en espacios públicos de México. Revista de Arquitectura, 23(2), 24-35. https://doi.org/10.14718/RevArq.2021.3896

\author{
Elizabeth Sedano \\ Licenciatura en arquitectura, Tecnológico Nacional de México. Unidad \\ Académica El Grullo (México). \\ Maestría en Arquitectura Sostenible y Gestión Urbana. \\ (D) https://orcid.org/0000-0003-1677-6658 \\ https://scholar.google.com/citations?user=ULM_IOQAAAAJ\&hl=en \\ (19) g204600I@colima.tecnm.mx \\ (10) sedano.hdez@gmail.com \\ Peter Chung Alonso \\ Licenciatura en Arquitectura, Universidad de Guadalajara (México) \\ Maestría en Arquitectura Sostenible y Gestión Urbana, Universidad de \\ Colima (México). \\ (i) https://orcid.org/0000-0002-3724-1938p \\ (1) peter.chung@colima.tecnm.mx \\ (10) arq.pcarq@gmail.com \\ María Silvia del Rocío Covarrubias Ruesga \\ Licenciado en Arquitectura, Universidad de Guadalajara (México). \\ Maestría en Arquitectura, Universidad de Colima (México). \\ Doctorado en Ciudad, Territorio y Sustentabilidad, Universidad de las Pal- \\ mas de Gran Canaria (España). \\ (i) https://orcid.org/0000-000I -9120-7098 \\ (10) maria.covarrubias@colima.tecnm.mx \\ (10) rociocov@hotmail.com
}

\section{Resumen}

La dialéctica del espacio y la justicia es una temática surgida desde las culturas clásicas; a pesar de ello, no hay una definición establecida para el concepto de la justicia espacial, y cada autor lo aborda desde diferentes perspectivas, escalas y enfoques, a partir del entorno que lo rodea. Por ello, el propósito de este trabajo radica en el análisis de la justicia espacial, desde la conceptualización de un marco referencial desarrollado por investigadores asiáticos, con el objetivo de encontrar la aplicabilidad de este término en los espacios públicos de México. Este artículo surge de la revisión de literatura a través de medios digitales (buscadores académicos como Google Scholar y Redalyc), para inferir los componentes que resaltan la vivencia de la justicia espacial en el país. Los resultados muestran que hay componentes que, por su magnitud y su alto impacto, deberían ser analizados como factores primordiales, como en el caso de la seguridad, la privatización del espacio público y la accesibilidad universal. Se concluye con la generación un marco conceptual que integra teóricamente los factores determinantes a escala nacional para el análisis de la justicia espacial, y se sugiere, como seguimiento a la línea de investigación, crear nuevos instrumentos aplicados a espacios públicos específicos dentro del país.

Palabras clave: derecho a la ciudad; espacio público; integración social; justicia social; seguridad ciudadana

\begin{abstract}
The dialectic of space and justice is a theme that has emerged from classical cultures. Despite this, there is no established definition for the concept of spatial justice, and each author approaches it from different perspectives, scales and approaches, based on the surrounding environment. Therefore, the purpose of this paper lies in the analysis of spatial justice from the conceptualization of a referential framework developed by Asian researchers to find the applicability of this term in public spaces in Mexico. This paper arises from the literature review through digital media (academic search engines such as Google Scholar and Redalyc) to infer the components that highlight the experience of spatial justice in the country. The results show that there are components that, due to their magnitude and high impact, should be analyzed as primary factors, as in the case of security, privatization of public space and universal accessibility. It concludes with the generation of a conceptual framework that theoretically integrates the determining factors on a national scale for the analysis of spatial justice, suggesting as a follow-up to the line of research, the creation of new instruments applied to specific public spaces within the country.
\end{abstract}

Keywords: citizen security; public space; right to the city; social integration; social justice Recibido: febrero 2/2021

\section{Introducción}

Este artículo se deriva del proyecto académico de posgrado Actualización del Manual de Procesos Participativos para el Diseño de Espacios Públicos del municipio de Colima integrando mecanismos de inclusión social, que se desarrolla en el programa de la Maestría en Arquitectura Sostenible y Gestión Urbana del Instituto Tecnológico de Colima, México. Adicionalmente, surge a partir de la contextualización de la problemática; es decir, para lograr la inclusión social en el espacio público debe respetarse el derecho a la ciudad y, con ello, lograr la justicia espacial. Sin embargo, se identificó que, como tal, no existe un referente teórico aplicable a los espacios públicos de México.

La justicia espacial es un concepto presente en la geografía, la sociedad, la economía, el ambiente, la política, la innovación y el arte, que no cuenta con una definición única (Soja, 2009). Surge a partir del derecho de la ciudad a dar una ordenanza en contra de injusticias sociales aplicadas a la urbe, como la discriminación, la inseguridad y la segregación (Ziccardi, 2019). Debido a la falta de una definición exacta, el término se ajusta y se adapta en relación con el contexto en el que se desarrolle, la configuración del espacio o la percepción de los usuarios, así como la intensidad con la que estos últimos pueden apropiarse de su entorno. 
En lo referente a espacios públicos abiertos, Jian et al. (2020) proponen un marco conceptual para el análisis de la justicia espacial, definido por cinco factores fundamentales: 1) el acceso y el mantenimiento; 2) la sociabilidad y la diversidad; 3) la interacción social 4) la demanda y la provisión, y 5) el estrato social y la información. Considerando la subjetividad de los parámetros de la justicia espacial, los mismos autores sugieren que su marco puede ser adaptable para responder a las características del entorno donde se lo implante.

Los espacios públicos en México desempeñan un rol histórico, debido al impacto que tienen en los ciudadanos; son áreas donde surge lo común, en las que se manifiestan la cultura y la tradición a través de la apropiación (Ribera, 2019); sin embargo, actualmente estos espacios enfrentan problemáticas socioespaciales, entendidas como injusticias: la privatización, la inseguridad y el abandono. Por ello, se considera necesario definir los factores que permitan el análisis de la justicia espacial en el ámbito nacional, y que así puedan generarse a posteriori instrumentos aplicables en áreas públicas de regiones específicas.

Debido a lo anterior, este trabajo tiene como objetivo el estudio del marco conceptual base de Jian et al. (2020), y la revisión documental de literatura referente a los cinco factores ya mencionados. El análisis realizado es de carácter interpretativo, y concluye con la adaptación de elementos para integrar un nuevo orden de factores y componentes que definan teóricamente la aplicabilidad de la justicia espacial en México.

\section{Justicia y espacio}

La justicia es un concepto que ha existido desde las culturas clásicas, y que se ha transformado con el paso del tiempo y del entorno, para ajustarse a la situación, la cultura e, incluso, la geografía. Desde la antigua Grecia se planteaba que para la justicia había dos vertientes relevantes: una de ellas, referente a la observancia y el cumplimiento de la ley; otra, sobre la equidad en relación con la interacción de individuos, debido a que vivimos en sociedades (Pirie, 1983). Así, la justicia no se asocia a lo individual, sino que integra a un tercero; se refiere a la conjugación entre seres generando el concepto de justicia social (Sarmiento et al., 2019).

En 1971, Rawls propuso como teoría que la justicia es un término referente a la equidad, en el que la sociedad actúa en conjunto y opta, por sus medios, por lo que percibe como una igualdad (Orum et al., 2019), de manera que lo justo se asocia directamente a la percepción de los individuos en lo referente a la colectividad. Para autores como Harvey (1992), este concepto tiene toda una familia de significados, ya que encaja en diferentes léxicos a partir del juego de palabras que se utilicen para definirlo.
Aunado a lo anterior, la justicia se relaciona con un derecho que puede ser objetivo cuando se lo observa a través de una situación específica, y subjetivo, cuando dicha situación es vista con una perspectiva individual (Sarmiento et al., 2019). Esta dialéctica se modifica en relación con cada situación, cada sociedad, cada cultura y cada geografía; es decir, la justicia es un elemento abarcador que requiere la delimitación de parámetros para su análisis.

Como contraparte, hay autores que sostienen que es mucho más factible analizar lo injusto - debido a la abstracción y la utopía que abarca el término justicia-, por cuanto es una condición tangible y objetiva que en más de una ocasión provoca la movilización social para su abolición (Salamanca et al., 2019).

Por otro lado, el espacio es un cúmulo de elementos materiales que surgen como producto de diferentes procesos sociales del humano (Castañeda et al., 2016). El uso como tal del espacio no ocurre de forma espontánea en el vacío, sino que sucede a través de la explotación del espacio físico para dar origen a diferentes configuraciones en las que convergen los usuarios (Páramo \& Burbano, 2014). La utilización del espacio genera áreas donde los individuos se desarrollan y crean historia. Así, los espacios construidos forjan una importancia simbólica y una cultura de ocupación natural (Ribera, 2019).

Lo anterior sugiere que la justicia se logra a partir de la apropiación del espacio en sus diferentes usos. Es aquí donde entran los imaginarios urbanos, ya que hacen referencia a la percepción que tienen los usuarios sobre la ciudad (Silva, 2006): el imaginario es un elemento subjetivo que cambia entre cada individuo; dicho de otra forma, el usuario se apropia del espacio según la percepción que tenga de este, le da uso y significado dependiendo de su visión, y por eso las ciudades deben ser analizadas, en cuestión de justicia, no solo desde un enfoque político o uno económico, sino también, el de la perspectiva que genera cada individuo (Bravo, 2010). Cada percepción, cada uso y cada apropiación del espacio es diferente y subjetiva desde la individualidad, y esto tiene impacto directo con la interpretación de la justicia.

\section{La justicia espacial}

No existe un concepto establecido que defina la justicia espacial, sino que se adapta y ajusta su léxico según la escala y el contexto en el que se implante. La justicia espacial hace referencia a la justicia social dentro de un determinado espacio (Pirie, 1983); surgió a partir de los años ochenta del siglo XX y se ha vuelto una base teórica para proyectos cuyo fin es el bienestar social (Hidalgo, 2018). Se relaciona con las necesidades de los ciudadanos en cuanto al derecho a habitar (Borja, 2019), a la vivienda digna (Calderón, 2019), 
al disfrute de los espacios públicos (Carrión, 2019), la manifestación para buscar nuevos derechos (Segovia \& Jordán, 2005) y, en general, a ser partícipes de la ciudad a través de lo común (Chatterton, 2010) en cualquier escala geográfica (Soja, 2009). Los principales exponentes de la temática tienen diversas definiciones de este concepto (Iveson, 2011), que concluyen con la misma premisa: "No puede haber ciudadanos de segunda clase" (Thiébaut, 2011, p.136).

La justicia espacial se busca a partir de movimientos sociales que exponen y debaten las injusticias percibidas por los ciudadanos en su cotidianidad (Hernández, 2017), y que, a partir de la reflexión de estas patologías, buscan la transformación del espacio (Salamanca et al., 2019). Es un concepto que va más allá de lo que las leyes norman, pues refleja la posibilidad real que tienen los usuarios para apropiarse (o no) del entorno (Pérez, 2004). Para algunos autores, es un término que podría asemejarse a la ciencia ficción, debido a la idealización de los imaginarios urbanos: vivimos el espacio que percibimos (Castrillo, 2017).

Se trata de un concepto adaptable, debido a que sus componentes difieren en relación con cada espacio e, incluso, en cada región geográfica (Ziccardi, 2019), pues, por ejemplo, los problemas sociales que afectan a Noruega no corresponden a aquellos surgidos en México por motivos culturales, geográficos y económicos. Y a pesar de ello, existen elementos que pueden ser homogeneizados cuando tratan la misma problemática desde diferentes visiones: hablar de justicia espacial significa hablar del derecho.

La teoría del derecho a la ciudad es propuesta por Henri Lefebvre (1972) y David Harvey (2008), apoyados ambos en teorías marxistas en oposición al capitalismo, con una perspectiva desde la clase obrera, a los llamados desposeídos, a quienes se les negaba la apropiación del espacio tanto público como de vivienda; de estos exponentes surge la iniciativa de visualizar la justicia espacial desde la planeación de políticas públicas que mejoraran la calidad de las relaciones sociales de los ciudadanos vulnerables (Garnier, 2017). Estas protestas a partir de la identificación de lo injusto incitan a los usuarios a apropiarse del espacio para exigir la creación de su derecho; Cuberos y Díaz (2018) aseguran que en ese momento es cuando la verdadera apropiación ocurre.

La Carta Mundial por el Derecho a La Ciudad tiene como su objetivo principal promover ciudades que garanticen el goce integral de sus habitantes en cuanto a economía, cultura y utilización de recursos; todo, encaminado a lograr la justicia y la equidad (Marques, 2005). En dicha carta se define una serie de elementos que debe integrar una ciudad para ser justa. Por su parte, ONU-Hábitat (2020) define los siguientes componentes como necesarios para definir el derecho a la ciudad:
1. Ciudades libres de discriminación

2. Ciudades con igualdad de género

3. Ciudades para todas las personas

4. Ciudades participativas

5. Ciudades accesibles y asequibles

6. Ciudades con espacios y servicios públicos de calidad

7. Ciudades con economías diversas e inclusivas

8. Ciudades sostenibles con vínculos urbano-rurales

De lo anterior se entiende que la ciudad está compuesta por diferentes espacios configurados a diversas escalas, y la relación de justicia que tienen estas áreas en conjunto con la ciudadanía se refleja en la aplicación del derecho que tienen los usuarios para su goce y su apropiación.

Por otro lado, resulta interesante analizar la teoría de la justicia espacial de Edward Soja (2009), ya que esta aborda tres ejes o factores que rigen este concepto:

a) La espacialidad ontológica del ser

b) La producción social de la espacialidad

c) La dialéctica socioespacial

El primero de estos factores hace referencia al hecho de que todos los seres ocupamos un espacio: 1) uno físico, debido a que somos seres y existimos como tal, y 2) otro social, debido a que estamos inscritos en comunidades, y aun cuando somos temporales, somos parte de un entorno tanto físico como social. El segundo factor se refiere a cómo el espacio es producido para abastecer las necesidades de sus usuarios: generamos el espacio que necesitamos dependiendo de las características que requerimos de este, y por otro lado, al ser sociales, nos apropiamos del espacio y le damos uso de acuerdo con nuestras necesidades. Para el tercer factor, el autor hace referencia a cómo damos forma a la sociedad a partir de los espacios, y viceversa.

Producimos espacios públicos de acuerdo con las necesidades sociales generando interconectividad en la trama urbana. En este punto es interesante retomar la teoría de Carrión (2019), la cual sugiere que estas áreas no son simplemente superficies para ocupar, sino toda una relación social.

\section{La dialéctica de la justicia espacial con el espacio público}

El espacio público es el área donde convergen diferentes usuarios para abastecer sus necesidades en colectividad; principalmente, las de recreación o de servicio (Briceño, 2018). Son sitios que las personas ocupan, donde circulan de manera continua en su día a día, lugares en común dentro de una sociedad (Fonseca, 2014), donde se forma, ocurre y evoluciona la historia de las ciudades (Bojórquez et al., 2019), una materialidad que estructura al sistema urbano (Pinzón \& Echeverri, 2010), fundamental al construir y ordenar 
el territorio a través de sus usos y sus actividades (Pérez, 2004).

La justicia espacial dentro del espacio público abierto busca la democratización a partir de la apropiación (Páramo \& Burbano, 2014). Hace referencia a los espacios donde los usuarios pueden desarrollarse sin importar su género, sus ingresos, si se encuentran en situación de discapacidad, o cualquier otro limitante que provoque la segregación o la discriminación de individuos, ya que el espacio público es para todos (Valera, 1999). El reordenamiento del espacio social y la solución a las problemáticas del espacio público promueven la intervención de la política (Cuberos \& Díaz, 2018), la cual se convierte en uno de los agentes de mayor relevancia para el impulso de la justicia espacial. Para su análisis existen diferentes componentes que integran la eficiencia espacial, ya que un espacio deficiente genera injusticia (Hernández, 2017). En esta investigación se observa y se estudia el marco conceptual propuesto por Jian et al. (2020) para la planeación de espacios públicos abiertos, el cual, a través de un análisis exhaustivo de fuentes y cuestionarios aplicados a escolares y expertos, define cinco factores que generan la justicia espacial y sus componentes, como se expone en la tabla 1.

Este marco conceptual sugiere que los cinco factores propuestos son los más relevantes al momento de analizar la justicia espacial en espacios públicos abiertos; sin embargo, sus autores sugieren que los componentes que lo integran pueden ser adaptados y ajustados al contexto en el que se desarrollen.

En cuanto al debate sobre el espacio público en América Latina, diferentes autores sugieren que en estas áreas se desarrolla la democracia a partir de la opinión pública, debido a que los usuarios se perciben como libres en lo común (Ramírez, 2015), y los definen como espacios de diversa apropiación donde se generan colectivos socioculturales (Bojórquez et al., 2019).

Para Carrión (2019), estos espacios que, en teoría, deberían ser integradores de la trama urbana, se enfrentan a problemáticas como:

- La fragmentación de las ciudades, debido a que la falta de articulaciones entre sus espacios provoca la alienación de los usuarios que no se reflejan con el entorno.

- La segmentación social generada a partir de la privatización de espacios. En este punto, los espacios públicos se vuelven exclusivos para las diferentes clases sociales y, por ende, difícilmente se genera inclusión.

- La difusión urbana, provocada por la desarticulación de las ciudades, lo cual, en vez de promover cohesión social, la difumina de manera que no pueda ser percibida por los ciudadanos.

\begin{tabular}{|c|c|}
\hline Factor & Componentes \\
\hline \multirow{7}{*}{ F1. Acceso y mantenimiento } & Accesibilidad y proximidad \\
\hline & Seguridad \\
\hline & Caminable \\
\hline & Mantenimiento y manutención \\
\hline & Amenidad e instalaciones \\
\hline & Asequibilidad \\
\hline & Área de servicio \\
\hline \multirow{4}{*}{ F2. Sociabilidad y diversidad } & Actividad de soporte \\
\hline & Tipo de espacio \\
\hline & Sociabilidad \\
\hline & Publicidad \\
\hline \multirow{3}{*}{ F3. Interacción social } & Edad \\
\hline & Ingresos \\
\hline & Empleo \\
\hline \multirow{3}{*}{ F4. Demanda y provisión } & Número \\
\hline & Área \\
\hline & Medida \\
\hline \multirow{2}{*}{ F5. Estrato social e información } & Participación y democracia \\
\hline & Información y conocimiento \\
\hline
\end{tabular}

- La inseguridad, que provoca miedo en los usuarios y el rechazo al área pública.

- La privatización del espacio, donde ya no existe área pública para coexistir, sino solo una serie de espacios privados.

\section{Aplicabilidad de la justicia espacial en los espacios públicos de México}

En México, la dialéctica del espacio público gira en torno a dos elementos: 1) la función práctica y 2) la historia. Estas áreas se cargan de efímera versatilidad para darles distintos usos, ya sean tianguis (comercio al aire libre), ferias, eventos culturales, religiosos, escolares o deportivos, o simplemente, para disfrutar del exterior; de ahí que los principales espacios públicos abiertos para los mexicanos sean las alamedas y las plazas mayores (Ribera, 2019).

México es un país cargado de imaginarios debido a su diversidad cultural (Goytia \& Martínez, 2019), los espacios públicos se reinventan y transmiten la apropiación, generan un incentivo histórico al adecuarse a las necesidades del usuario y se convierten en testigo primordial para la evolución de las ciudades; sin embargo, lo público se ve amenazado por elementos determinantes que logran restringir el espacio a través del turismo (Bojórquez et al., 2019), la privatización (Ramírez, 2015), la violencia y la inseguridad (Jasso, 2015).

Aunado a lo anterior, el avance de la globalización amplificó estas amenazas: su impacto en los espacios públicos causó problemas que aún buscan solución: por un lado, áreas públicas tradicionales, como las calles y las plazas, fueron privatizadas o abandonadas, lo cual provocó que el tipo de usuarios que se apropian de ellos
A Tabla 1. Factores y componentes que integran el marco conceptual para la planificación de espacios públicos abiertos. Fuente: elaboración propia, con base en Jian et al. (2020). 
se reduzca. Además, los espacios públicos de carácter histórico y patrimonial se enfocan cada vez más en turistas e individuos pertenecientes a un estrato socioeconómico alto (Giglia, 2002).

Por otro lado, cuando estas áreas se convierten en vacíos dentro de la trama urbana promueven la inseguridad, y con ello provocan agorafobia en los usuarios; este miedo al espacio surge de la percepción del usuario no solo por la cantidad de delitos que se hayan cometido en él, sino, también, por carencias en el mantenimiento y la infraestructura, así como la falta de iluminación (Jasso, 2015)

En el país, de hecho, destaca de forma significativa la inseguridad enfocada en una situación de género, donde la violencia hacia la mujer en espacios públicos tiene alto impacto a partir de irregularidades como acosos, violaciones y secuestros (Soto, 2012); de lo anterior es que han surgido, con el paso del tiempo, distintos movimientos sociales que buscan transformar el sistema político para lograr esa justicia espacial, y con ello garantizar el derecho a la ciudad (Aguilar, 2010).

Entre la legislatura enfocada en el cumplimiento del derecho a la ciudad y, por ende, en la justicia espacial en México destacan:

- El artículo $1^{\circ}$. de la Constitución Política de los Estados Unidos Mexicanos (1917), ya que engloba el hecho de que, como sociedad, podemos gozar de los Derechos Humanos.

- La Carta de la Ciudad de México por el Derecho a La Ciudad, la cual se desprende directamente de su versión con aplicabilidad mundial (Sánchez, 2018).

- La Ley General de Desarrollo Social (2004) aborda las acepciones de justicia en su artículo $3^{\circ}$, haciendo hincapié en la justicia socioespacial y en la calidad de vida de los ciudadanos.

- La Ley General de Asentamientos Humanos (2020), que plantea de forma legal el derecho a la ciudad, a través de la cohesión social, la equidad y la inclusión eliminando situaciones como la discriminación, la segregación y la marginación, y promoviendo así el desarrollo social a cualquier escala.

Cuando se desarrollan nuevos espacios públicos sin un análisis previo en relación con sus usuarios y su función, se generan áreas carentes de identidad, lo cual promueve problemáticas como la segregación social y la fragmentación de la ciudad; así, la justicia espacial se busca y se conquista a través de la política y del movimiento social que impulsa la promulgación de leyes que salvaguarden y protejan de forma equitativa la diversidad de usuarios.

\section{Metodología}

Partiendo de la hipótesis de que regionalizar los elementos de la justicia espacial generará una base teórica para su posterior análisis, se utilizó como referente el marco conceptual para la planificación de espacios públicos abiertos propuesto por Jian et al. (2020), donde a partir de cinco factores y sus componentes, referidos en la tabla 1, se estudió su relación y su aplicabilidad en México.

El marco conceptual utilizado como base fue elegido debido a su reciente creación y por el componente de innovación, lo cual permite, según los autores, que sus elementos sean modificables y adaptables a diferentes regiones geográficas para completarlo.

Para cada uno de los cinco factores originales se asignaron de forma inferencial indicadores que permitieran medir sus componentes. Algunos índices se repiten debido a la relación intrínseca de elementos.

El primer factor (acceso y mantenimiento) es el de mayor extensión; consta de siete componentes. Los principales indicadores relacionados hacen referencia a la privatización, la accesibilidad universal y la inseguridad. El último componente (área de servicio) es demasiado específico, por lo cual se consideró que asignar un índice sería redundante.

F1. Acceso y mantenimiento:

- Accesibilidad y proximidad

- Privatización

- Espacios fuera de la mancha urbana

- Movilidad

- Seguridad

- Índice de delincuencia y sentimiento de seguridad

- Transitable

- Accesibilidad universal

- Características del espacio

- Mantenimiento y manutención

- Accesibilidad universal

- Equipamiento

- Amenidad e instalaciones

- Confort

- Habitabilidad

- Asequibilidad

- Privatización

- Área de servicio

El segundo factor base (sociabilidad y diversidad), consta de cuatro componentes. Los indicadores asignados corresponden a elementos de apropiación y uso del suelo, pues se infiere que de esta forma encuentran aplicabilidad en México. Denota, nuevamente, la presencia de la privatización como indicador para el componente de la publicidad. La sociabilidad se considera elemento específico no medible, por lo que no se asigna un índice. 
F2. Sociabilidad y diversidad:

- Actividad de soporte

- Recreación y cultura

- Actividades económicas

- Tipo de espacio

- Características del espacio

- Sociabilidad

- Publicidad

- Privatización

El factor 3 (interacción social) está integrado por componentes específicos, que corresponden a edad, ingresos y empleo. Para estos elementos específicos se consideró oportuno vincularlos entre sí para definir como indicador la caracterización de usuarios.

\section{F3. Interacción social:}

- Edad, ingresos y empleo

\section{- Caracterización de usuarios.}

Por su parte, el factor 4 corresponde a demanda y provisión, donde los elementos que lo componen son nuevamente considerados específicos y no se considera un indicador aplicable a ninguno de ellos.

F4. Demanda y provisión:

- Número

- Área

- Medida

Finalmente, en el factor 5 (estrato social e información) los índices para determinar sus componentes son más abarcadores; principalmente, el de democratización de espacios. Se consideró manejarlo de esta forma, debido al alcance del presente trabajo, donde cada índice otorgado propone una base teórica para el análisis de componentes. Por su parte, el último componente es, nuevamente, específico, y puede vincularse directamente como un indicador.

F5. Estrato social e información:

- Participación y democracia

- Democratización de espacios

- Participación ciudadana

- Información y conocimiento

Una vez definidos los indicadores, se llevó a cabo la búsqueda de literatura, donde los índices definidos presentaron un referente para localizar información específica aplicable de forma general al área de estudio. La recolección de información se hizo primero con enfoque en América Latina (para homogeneizar el área de estudio), y una segunda revisión enfocada directamente en lo aplicable a México (como área específica de análisis).

Las herramientas para la recolección de datos fueron digitales, utilizando los siguientes buscadores académicos: Google Scholar, Scielo, Dialnet y Redalyc, así como la plataforma de Research Gate. Es necesario destacar que en algunas de las referencias primarias no fue posible acceder de forma gratuita al documento digitalizado por ningún medio, por lo cual se buscaron otros trabajos de los mismos autores que pudieran relacionarse con la temática principal.

Con la información recabada se procedió al siguiente paso, en el que se generó una matriz correlacional para organizar los cinco factores. A cada uno de sus componentes se les anexó una breve definición, y en otra columna, los índices previamente definidos. La última columna de la matriz vincula los indicadores con los autores que abordan la temática enfocados directamente en las teorías de la justicia espacial y el derecho a la ciudad. Los componentes que no encontraron indicadores por sus características también fueron considerados en la recolección de literatura.

Del análisis de la matriz completada se generó un código de colores para identificar visualmente su correlación o su impacto. Se detectaron elementos que entrelazaban sus indicadores con factores diferentes, por lo cual se asignó el color azul para representar indicadores repetidos; el rojo y el amarillo se implementaron para identificar componentes que no guardan relación entre sí, y en los que se encontró información relevante con incidencia en México que sugiere un tratamiento especial para su análisis. Con lo anterior se detectaron los elementos específicos que sugieren la modificación del marco conceptual base para una aplicabilidad más acertada en el ámbito país.

Los componentes detectados de alto impacto y los que muestran relación entre sus componentes fueron modificados y reorganizados según su grado de incidencia a escala nacional, con lo que se generó la propuesta de reestructuración del marco conceptual.

En esta nueva propuesta se integró una columna para comparar y analizar si el marco definido cumplía con los elementos de la justicia espacial y el derecho a la ciudad usando como referente lo establecido por organismos como ONU-Hábitat, la Coalición Internacional del Hábitat Latina y la teoría de Edward Soja (2009).

V Figura 1. Esquema metodológico.

Fuente: elaboración propia (2020).

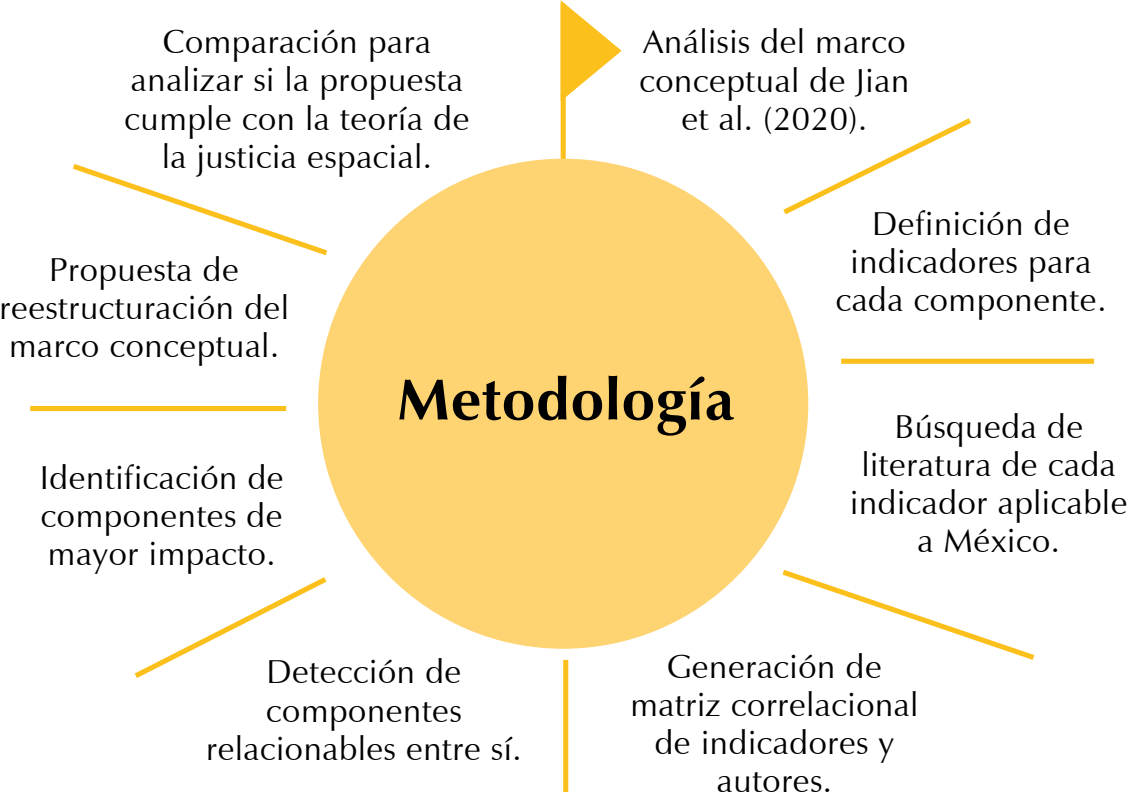


En la figura 1 puede apreciarse de forma sintetizada el esquema metodológico explicado. Iniciando en la pestaña de la parte superior, y siguiendo el sentido horario, se muestra el orden de las actividades realizadas, desde el análisis del marco conceptual de referencia, la generación de la nueva propuesta y la comparación con las teorías analizadas.

\section{Resultados}

Del marco conceptual para la planificación de espacios públicos abiertos (Jian et al., 2020) se analizaron los cinco factores y sus componentes, para regionalizarlos e identificar cuáles de ellos encuentran aplicabilidad en México. El marco propuesto sugiere una base teórica para el análisis de la justicia espacial de forma generalizada, como se muestra en la tabla 2.

Fue necesario analizar tanto los elementos teóricos de la justicia espacial como su contraparte para ampliar el rango de la información. Se trató como lo justo aquello promulgado por el derecho a la ciudad, y como lo injusto, la problemática generada en el área pública. De esta manera, para el caso de la apropiación se consideraron los trabajos referentes al abandono de espacios; para la participación ciudadana, aquellos que mencionaban el desinterés por parte de los usuarios, y sobre la tenencia del espacio, lo referente a la privatización.

En la definición de indicadores medibles con aplicabilidad en México, el código de colores generado por correlación e incidencia demuestra los factores de mayor impacto para el país. Este mismo orden de colores se extrapola en el nuevo marco propuesto para identificar visualmente las principales modificaciones realizadas.

Dentro del factor uno (acceso y mantenimiento), destaca el componente de seguridad, en el cual no solo se identificaron diversos autores que exponen su importancia, sino también, los datos recabados de la plataforma del Instituto Nacional de Estadística y Geografía (INEGI); se considera pertinente mencionar lo recuperado de la Encuesta Nacional de Seguridad Pública Urbana (ENSU), en la cual, para septiembre del 2020, un porcentaje del 52,4\% de la población de 18 años y más, señalaron percibir inseguridad en los parques recreativos, el 39,6\% han atestiguado incivilidades alrededor de su vivienda y el 61,8\% cambió de hábitos por temor a ser víctima de la delincuencia (INEGI, 2020a).

Lo anterior genera un análisis a partir de dos enfoques: 1) la percepción individual de los usuarios y 2) el impacto real en la cotidianidad del área pública; por otra parte, el porcentaje de mujeres que han actuado por agorafobia resulta mayor que el de los hombres, pues en cuanto a hostigamiento y violación sexual la encuesta más reciente del mismo instituto arrojó que el 91,8\% han sufrido manoseos, exhibicionismo e intentos de violación, y el 82,5\% sufrieron el delito de violación (INEGI, 2020b).

El análisis de información de esta índole sugiere que, para la justicia espacial, aplicada en el área pública de México, el principal factor por considerar es el de la seguridad, tanto percibida como detectada estadísticamente, por lo que este elemento no puede ser considerado un componente más dentro de la nueva propuesta.

En el mismo factor se encontraron indicadores vinculados entre sí, entre los que se destacan la privatización de espacios y la accesibilidad universal. Estos elementos fueron reflejados también en el factor dos, con el componente de publicidad, por lo que se consideró en la propuesta homogeneizarlos para la creación del factor denominado acceso.

El resto de los elementos del primer factor corresponden al mantenimiento del espacio y la amenidad. Para estos casos se contemplaron como indicadores el equipamiento, el confort y la habitabilidad del espacio. Aunque estos índices abarcan un campo de estudio extenso, se consideraron para este proyecto aquellos trabajos donde se vinculan de forma explícita estos conceptos con la justicia espacial, mientras que para el componente área de servicio no se encontró literatura relacionada con la temática principal.

Para el factor dos (sociabilidad y diversidad), los componentes que no se vinculan a otro factor son: actividad de soporte y tipo de espacio. Existe entre ellos una correlación identificada en la literatura recabada, de manera que fueron analizados dentro del mismo parámetro, al que se denominó configuración del espacio; por otro lado, del componente de la sociabilidad no se encontró información referente a la justicia espacial.

Para el factor tres (interacción social), se propone que sus componentes (edad, ingresos y empleo) sean medidos a través de la caracterización de usuarios. Este indicador aparece implícito en la teoría general sobre la justicia espacial, pero entre los autores que consideran primordial este elemento destacan Lefebvre (1972) y Harvey (2008), con las esferas sociales, así como Soja (2009), con el ser ontológico y su dialéctica de producción socio-espacial. La nueva propuesta de marco conceptual vincula la interacción social con la apropiación de la diversidad de usuarios en el espacio público.

El factor número cuatro (demanda y provisión) tiene como componentes el número, el área y la medida del espacio, para los cuales no se encontró literatura que los relacionara de forma específica con la justicia espacial; sin embargo, sus elementos son considerados en la propuesta a partir de las características intrínsecas del espacio.

Finalmente, en el factor número cinco (estrato social e información) se encuentran la participación y la democracia, así como la información y 


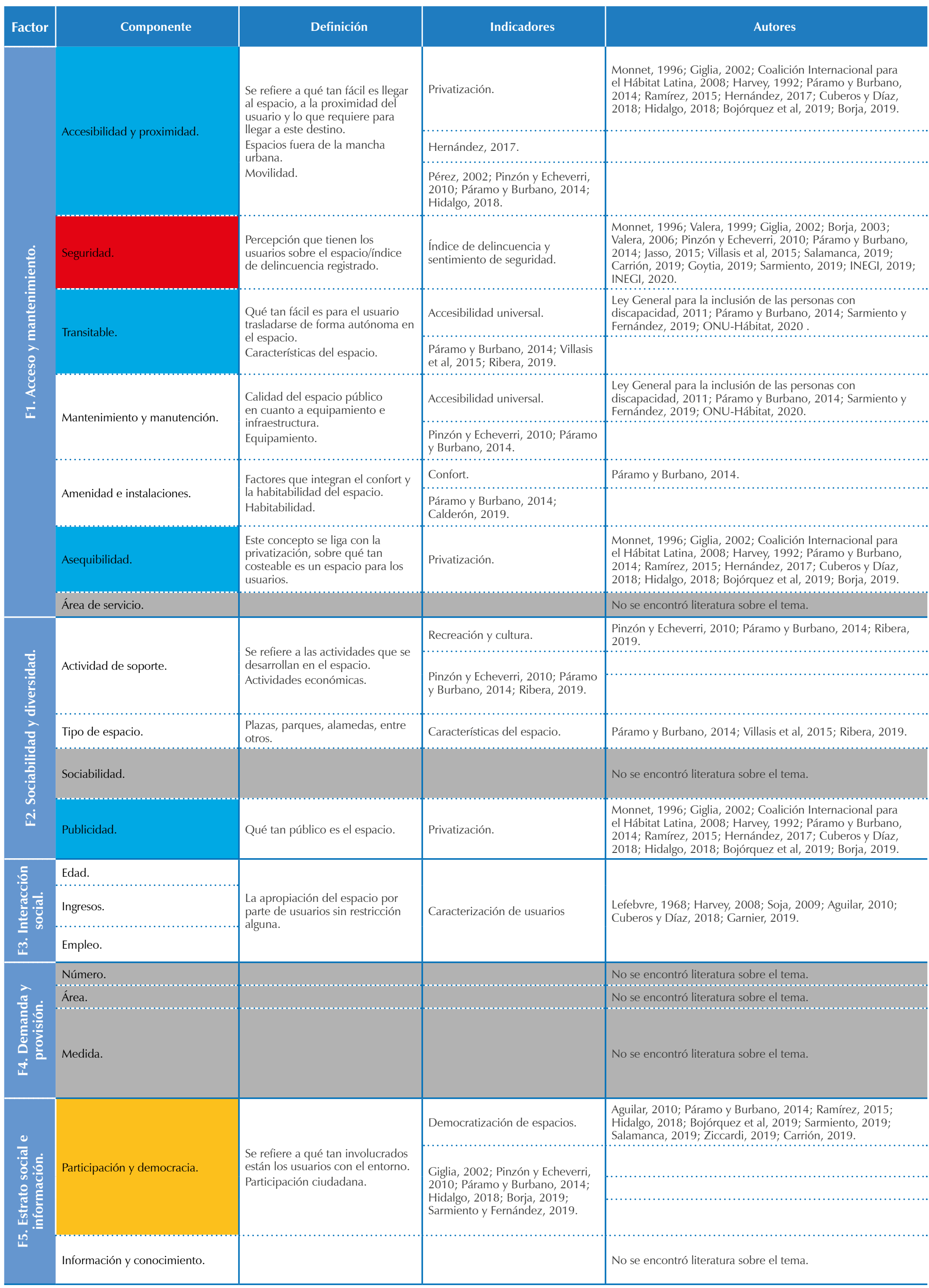


el conocimiento, como sus dos únicos componentes. Del primero se pueden separar ambos conceptos, y la información recabada demuestra que es un factor cuya importancia en materia de justicia espacial ha sido considerada por diversos autores; sin embargo, el segundo componente carece de literatura aplicable.

A partir de lo anterior, se generó una matriz para reestructurar el marco conceptual base, como se muestra en la tabla 3. La propuesta conserva la estructura de cinco factores, donde su rediseño contempla:

1. Factor 1: seguridad

2. Factor 2: acceso

3. Factor 3: mantenimiento

4. Factor 4: diversidad de usuarios

5. Factor 5: configuración del espacio

La propuesta sugiere como principal factor la seguridad, analizada tanto desde la percepción del usuario como desde los datos estadísticos registrados. Le sigue el factor de acceso, a partir de la restricción de los usuarios. El factor tres se refiere al mantenimiento, y no sufre muchos cambios en relación con el marco referencial base. Por su parte, el factor cuatro se destina a la diversidad de espacios a partir de la integración de estos. Finalmente, el factor cinco se destina a la configuración del espacio, donde se destacan la participación ciudadana para la democratización de espacios y la accesibilidad universal como característica implícita para lograr la justicia espacial.

Para el desarrollo de la matriz con esta nueva propuesta, en la primera columna se presentan los factores mencionados, siguiendo en su diseño el código de color resultante del análisis generado previamente. Se modificaron los componentes que por su impacto deben ser tratados como ejes determinantes, y se consideran dentro del mismo factor los que resultaron relacionables.

En la segunda columna se reorganizaron los componentes basados en el estudio de referencia y se propusieron unos nuevos para los factores recién integrados. La tercera columna se destinó para el anexo de una breve definición de cada componente, y de esta se desprenden los indicadores con los que se propone que sean medidos estos factores.

En lo referente a la última columna de observaciones, cuya función es relacionar con qué teorías y recomendaciones internacionales cumplen los conceptos presentados, se consideró lo establecido por la Coalición Internacional para el Hábitat Latina (2008) en cuanto a la construcción de espacios públicos seguros y de acceso equitativo para sus usuarios, así como lo relativo al derecho a la ciudad; se lo reforzó con los ocho componentes de esta misma temática del derecho, establecida por ONU-Hábitat, y que ya fueron mencionados en el marco teórico. Se relaciona, además, la teoría de la justicia espa- cial, elaborada por Edward Soja (2009), y en la que sintetiza el concepto a través de tres factores que interrelacionan al usuario con el espacio y la función de este.

\section{Discusión}

La justicia espacial es una característica mayormente relativa, que cambia según el contexto en el cual se la analice, pues sus factores se componen de elementos que trabajan sistemáticamente para funcionar. Reunir los conceptos que forman un marco para esta temática y adaptarlos a la situación de México implica ajustarlos a fin de enfocar las realidades del país.

Proponer el análisis de la justicia espacial a escala nacional es un tema extenso y complejo, debido a los conceptos ya expuestos. Para determinar esta justicia es necesario considerar la envolvente del espacio público y esclarecer su dialéctica con la ontología de los usuarios; sin embargo, esta propuesta busca analizar dentro del debate entre lo justo (y lo injusto) para delimitar el panorama en relación con las problemáticas nacionales.

Resulta paradójico cómo la justicia es notoria cuando se la analiza desde su contraparte para darle objetividad; sin embargo, esto vuelve integral el marco conceptual, debido a la fusión de ideas y teorías que componen una misma temática, lo cual, teóricamente, promoverá soluciones a futuro que permitan alcanzar la justicia espacial aplicables de forma específica a localidades y las ciudades dentro del país.

Del análisis de la información recabada, resulta imprescindible hacer un estudio que promueva la solución de espacios públicos a partir de la identificación y la exposición de las problemáticas expuestas. Se espera que a partir del marco conceptual propuesto se generen instrumentos que expongan cómo es que se vive la justicia en espacios públicos de México, y que de esta manera se dejen de normalizar las injusticias espaciales a las que está sometida el área pública en la actualidad.

El elemento de mayor impacto detectado en esta investigación es el de seguridad, lo cual sugiere que los espacios públicos en México son injustos, espacialmente hablando, debido a la inseguridad; así mismo, destacan la privatización surgida desde enfoques como el turismo y la discriminación, al igual que la accesibilidad.

México, siendo un país cargado de historia y cultura, es un claro ejemplo de que los espacios públicos están vivos debido a sus características y a los significados que plasmamos en ellos. Crear espacios públicos más justos busca promover una apropiación equitativa que impulse la cohesión social.

Por último, la literatura que pueda relacionarse de forma implícita con la justicia espacial es tan 


\begin{tabular}{|c|c|c|c|c|}
\hline Factor & Componentes & Definición & Indicadores & Observaciones \\
\hline \multirow{7}{*}{ F1. Seguridad. } & \multirow{6}{*}{$\begin{array}{l}\text { Índice de } \\
\text { delincuencia } \\
\text { registrada. }\end{array}$} & \multirow{6}{*}{$\begin{array}{l}\text { Datos registrados donde } \\
\text { se analice el nivel de } \\
\text { delincuencia ocurrida. }\end{array}$} & $\begin{array}{l}\text { Acoso. } \\
\text { Intento de violación. }\end{array}$ & \multirow{7}{*}{$\begin{array}{l}\text { Cumple con lo que } \\
\text { establece la Coalición } \\
\text { Internacional para el } \\
\text { Hábitat Latina (2008), en } \\
\text { cuanto a la construcción } \\
\text { de espacios públicos } \\
\text { seguros. }\end{array}$} \\
\hline & & & Violaciones. & \\
\hline & & & Robo. & \\
\hline & & & Secuestro. & \\
\hline & & & Vandalismo. & \\
\hline & & & Venta de drogas. & \\
\hline & Agorafobia. & $\begin{array}{l}\text { Percepción de inseguridad } \\
\text { en el espacio por parte de los } \\
\text { usuarios. }\end{array}$ & $\begin{array}{l}\text { Actividades y/o conductas que los usuarios } \\
\text { evitan por miedo en un espacio público. }\end{array}$ & \\
\hline \multirow{7}{*}{ F2. Acceso. } & \multirow{2}{*}{$\begin{array}{l}\text { Accesibilidad y } \\
\text { proximidad. }\end{array}$} & \multirow{2}{*}{$\begin{array}{l}\text { Qué tan fácil es llegar al } \\
\text { espacio. }\end{array}$} & Movilidad. & \multirow{7}{*}{$\begin{array}{l}\text { Cumple con los } \\
\text { componentes del } \\
\text { Derecho a la Ciudad } \\
\text { establecidos por ONU- } \\
\text { Hábitat (2020). }\end{array}$} \\
\hline & & & Espacios alejados de la mancha urbana. & \\
\hline & \multirow{5}{*}{ Privatización. } & \multirow{2}{*}{$\begin{array}{l}\text { El espacio es costeable para } \\
\text { cualquier usuario. }\end{array}$} & Costro de ingreso. & \\
\hline & & & Discriminación por ingresos. & \\
\hline & & & 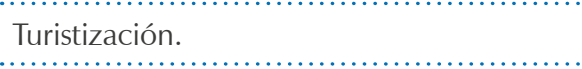 & \\
\hline & & $\begin{array}{l}\text { El usuario no es tratado como } \\
\text { extraño dentro del espacio. }\end{array}$ & Segregación. & \\
\hline & & & Apropiación selectiva. & \\
\hline \multirow{6}{*}{ F3. Mantenimiento. } & \multirow{4}{*}{$\begin{array}{l}\text { Mantenimiento del } \\
\text { espacio. }\end{array}$} & \multirow{4}{*}{$\begin{array}{l}\text { Se conserva la infraestructura } \\
\text { y equipamientos de forma } \\
\text { óptima. }\end{array}$} & $\begin{array}{l}\text { Mobiliario. } \\
\ldots \ldots \ldots \ldots \ldots \ldots \ldots \ldots \ldots \ldots \ldots \ldots\end{array}$ & \multirow{6}{*}{$\begin{array}{l}\text { Cumple los componentes } \\
\text { del Derecho a la Ciudad } \\
\text { establecidos por ONU- } \\
\text { Hábitat (2020). }\end{array}$} \\
\hline & & & $\begin{array}{l}\text { Iluminación. } \\
\text { n................ }\end{array}$ & \\
\hline & & & Infraestructura. & \\
\hline & & & Vegetación. & \\
\hline & \multirow{2}{*}{ Amenidad. } & \multirow{2}{*}{$\begin{array}{l}\text { Percepción del usuario sobre } \\
\text { el entorno. }\end{array}$} & Confort del usuario. & \\
\hline & & & Habitabilidad. & \\
\hline \multirow{7}{*}{$\begin{array}{l}\text { F4. Diversidad de } \\
\text { usuarios. }\end{array}$} & $\begin{array}{l}\text { Apropiación del } \\
\text { espacio. }\end{array}$ & $\begin{array}{l}\text { Cualquier usuario puede } \\
\text { hacer uso del espacio. }\end{array}$ & Caracterización de usuarios. & \multirow{7}{*}{$\begin{array}{l}\text { Cumple la teoría de la } \\
\text { justicia espacial de Soja } \\
\text { (2009) y los componentes } \\
\text { del Derecho a la Ciudad } \\
\text { establecidos por ONU- } \\
\text { Hábitat (2020). }\end{array}$} \\
\hline & \multirow{6}{*}{ Segregación social. } & \multirow{6}{*}{$\begin{array}{l}\text { Discriminación de cualquier } \\
\text { tipo. }\end{array}$} & Edad. & \\
\hline & & & Raza. & \\
\hline & & & Género. & \\
\hline & & & $\begin{array}{l}\text { Ingresos. } \\
\text {. }\end{array}$ & \\
\hline & & & Condición física. & \\
\hline & & & Otros. & \\
\hline \multirow{7}{*}{$\begin{array}{l}\text { F5. Configuración } \\
\text { del espacio. }\end{array}$} & \multirow{3}{*}{ Tipo de espacio. } & \multirow{3}{*}{$\begin{array}{l}\text { Características intrínsecas del } \\
\text { espacio público. }\end{array}$} & $\begin{array}{l}\text { Funcionalidad. } \\
\text {. }\end{array}$ & \multirow{7}{*}{$\begin{array}{l}\text { Cumple lo que establece } \\
\text { la Coalición Internacional } \\
\text { para el Hábitat Latina } \\
\text { (2008), la teoría de la } \\
\text { justicia espacial de Soja } \\
\text { (2009) y los componentes } \\
\text { del Derecho a la Ciudad } \\
\text { establecidos por ONU- } \\
\text { Hábitat (2020). }\end{array}$} \\
\hline & & & 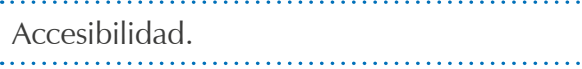 & \\
\hline & & & $\begin{array}{l}\text { Transitabilidad. } \\
\ldots \ldots \ldots \ldots \ldots \ldots \ldots \ldots \ldots \ldots \ldots \ldots \ldots \ldots \ldots \ldots \ldots \ldots \ldots\end{array}$ & \\
\hline & \multirow{3}{*}{$\begin{array}{l}\text { Actividades de } \\
\text { soporte. }\end{array}$} & \multirow{3}{*}{$\begin{array}{l}\text { Actividades que se realizan } \\
\text { en el espacio público. }\end{array}$} & $\begin{array}{l}\text { Actividades económicas. } \\
\text {. }\end{array}$ & \\
\hline & & & Actividades culturales. & \\
\hline & & & $\begin{array}{l}\text { Actividades deportivas. } \\
\text {. }\end{array}$ & \\
\hline & $\begin{array}{l}\text { Participación } \\
\text { ciudadana. }\end{array}$ & $\begin{array}{l}\text { Involucramiento de los } \\
\text { usuarios para el diseño e } \\
\text { intervención en los espacios } \\
\text { públicos. }\end{array}$ & Democratización de espacios. & \\
\hline
\end{tabular}

A Tabla 3. Propuesta de ordenamiento de factores para el análisis de la justicia espacial en México.

Fuente: elaboración propia, con base en la literatura recabada (2020). 
basta que no sería suficiente un artículo para integrarla, por lo que para este trabajo se tomaron como referencia aquellos que involucraban de forma específica la justicia espacial y el derecho a la ciudad.

\section{Conclusiones}

La presente investigación es meramente documental e inferencial. Bajo estos parámetros se logró adaptar los factores integradores de la justicia espacial definidos en el marco conceptual base de Jian et al. (2020), tras el análisis teórico de sus componentes aplicables en espacios públicos de México. El estudio referencial elegido fue considerado debido a que en su estructura integra los elementos propuestos por Edward Soja en su teoría sobre la justicia espacial y, a su vez, encuentran relación con el derecho a la ciudad.

Para el caso de México, los factores propuestos para el análisis de la justicia espacial integran la seguridad, el acceso, el mantenimiento del espacio, la diversidad de usuarios y su configuración. De estos factores se desprenden diferentes componentes e indicadores que corresponden no solo a trabajos previamente estudiados, sino a aquellas áreas que deben estudiarse y analizarse, por parte de todos los agentes involucrados, para la generación de espacios públicos.

El alcance del presente estudio se limita a la propuesta conceptual; sin embargo, las investigaciones futuras que aborden esta teoría conformarán resultados que permitan unificar criterios de justicia espacial, aplicados a posteriori a un sistema a escala nacional.

Analizar los factores expuestos en la tabla 2 para poder reajustarlos con la propuesta generada en la tabla 3 nos demuestra la complejidad que implica adaptar conceptos de importancia universal a las necesidades y el contexto en diferentes escalas geográficas. Cabe destacar que la propuesta de este trabajo busca generar no un instrumento único que permita visualizar la jus- ticia espacial, sino la creación de múltiples estudios que se integren dentro de la misma teoría, pero correspondan a las complejidades intrínsecas de cada trama urbana.

Para lograr la justicia espacial es necesario exponer las injusticias espaciales que sufren los ciudadanos en su relación con el espacio público. Se considera importante dejar de normalizar las problemáticas para generar espacios justos y equitativos, ya que cuando los espacios son apropiados por la delincuencia y la inseguridad la percepción del usuario se traduce en miedo; cuando carecen de accesibilidad universal se priva la apropiación, lo que vuelve al espacio ajeno, cansado e impropio; la segregación segmenta la ciudad, mientras que las áreas carentes de confort y mal planteadas limitan su utilización.

Este trabajo sugiere exponer primeramente la situación actual en cuanto a justicia espacial del país. Se propone la elaboración de un diagnóstico de los temas aquí tratados para validar el comportamiento de los factores propuestos buscando promover en todo momento el cumplimiento del derecho a la ciudad.

El marco propuesto integra elementos que resultan primordiales en el ámbito nacional, a partir de la información recabada; sin embargo, eso no significa que sea único o estático: por el contrario, es una propuesta flexible que se puede ajustar dependiendo del espacio físico por analizar. Pueden integrarse nuevos componentes y cambios en los indicadores, teniendo como fundamento la justicia espacial y el derecho a la ciudad.

\section{Contribuciones y agradecimientos}

Agradezco de forma especial a los coautores de este trabajo: el maestro Peter Chung y la doctora Rocío Covarrubias, por su apoyo y su contribución en el análisis y el ordenamiento de la información recabada. Su guía para convertir una idea, darle forma y traducirla en un trabajo real resultó imprescindible para lograr este artículo.

\section{Referencias}

Aguilar Sánchez, M. (2010). El espacio público y los movimientos sociales: reflexiones en torno a tres estudios de caso. En ¿Qué tan público es el espacio público en México? (pp. 297310). Editorial Fondo de Cultura Económica. https://www.uv.mx/blogs/accioncolectiva/ files/2015/10/EspacioPublico.pdf

Bojórquez Luque, J., Ángeles Villa, M. \& Gámez, A. E. (2019). El derecho a la ciudad y rescate del espacio público en zonas urbanas turistizadas. Aposta. Revista de Ciencias Sociales, (80), 109-128.

https://doi.org/http://www.apostadigital. com/revistav $3 /$ hemeroteca/ num80completo.pdf\#page $=109$

Borja, J. (2019). Ciudadanía, derecho a la ciudad y clases sociales. O la Democracia versus el Derecho. En F. Carrión Mena \& M Dammert Guardia (Eds.), Derecho a la ciudad, una evocación de las transformaciones urbanas en América Latina (pp.25-59). Editorial CLACSO.

http://biblioteca.clacso.edu.ar/clacso/ gt/20200519104921/Derecho-a-la-ciudad. pdf\# page $=61$

Bravo, B. [Armando Silva]. (diciembre, 2010). Imaginarios Urbanos. Research Gate. https://www.researchgate.net/ publication/48072525_Imaginarios_ urbanos/stats

Briceño Ávila, M. (2018). Paisaje urbano y espacio público como expresión de la vida cotidiana. Revista de Arquitectura, 20(2), 10-19. https://doi.org/10.14718/revarq.2018.20.2.1562

Calderón Cockburn, J. (2019). El derecho a la ciudad en América Latina y los enfoques sobre la ciudad informal. En F. Carrión Mena \& M. Dammert Guardia (Eds.), Derecho a la ciudad, una evocación de las trans- formaciones urbanas en América Latina (pp.95-110). Editorial CLACSO.

http://biblioteca.clacso.edu.ar/clacso/ gt/20200519104921/Derecho-a-la-ciudad. pdf $\#$ page $=61$

Carrión, F. (2019). El Espacio público es una relación no un espacio. En F. Carrión Mena \& M. Dammert Guardia (Eds.), Derecho a la ciudad, una evocación de las transformaciones urbanas en América Latina (pp. 191219). Editorial CLACSO. http://biblioteca. clacso.edu.ar/clacso/gt/20200519104921/ Derecho-a-la-ciudad.pdf\# page $=61$

Castañeda López, E. I., Alcántara Hernández, L. \& García Rivera, T. M. (2016). La construcción de otras mujeres y de otros espacios: el caso de San Miguel Teotongo. Bitácora Arquitectura, (33), 32-39.

http://dx.doi.org/10.22201/fa.14058901p.2016.33.57257 
Castrillo Romón, M. (2017). ¿Geohistoria o geoficción? Ciudades vulnerables y justicia espacial. Ciudades, (13), 245-248.

h t t p s://d o i.org/10.24197/ ciudades.13.2010.245-248

Chatterton, P. (2010). Seeking the urban common: Furthering the debate on spatial justice. City, 14(6), 625-628.

https://doi.org/10.1080/13604813.2010.52 5304

Coalición Internacional para el Hábitat Latina Oficina Regional para América. (2008). La carta mundial por el derecho a la ciudad. https://hic-al.org/que-hacemos/derecho-ala-ciudad/

Constitución Política de los Estados Unidos Mexicanos (CPEUM). (1917, 5 de febrero). Artículo 1.

Cuberos Gallardo, J. \& Díaz Parra, I. (2018). Justicia socio-espacial y disputa por los espacios centrales en el barrio del Abasto (Buenos Aires). Contexto, 12(16), 13-31. http://contexto.uanl.mx/index.php/contexto

Fonseca Rodríguez, J. M. (2014). La importancia y la apropiación de los espacios públicos en las ciudades. Paakat: Revista de Tecnología y Sociedad, (7).

http://www.redalyc.org/articulo. oa?id $=499051556003$

Garnier, J. P. (2017). El derecho a la ciudad desde Henri Lefebvre hasta David Harvey. Entre teorizaciones y realización. Ciudades, 15(15), 217-225.

ht tps://doi.org/10.24197/ ciudades.15.2012.217-225

Giglia, A. (2002). Privatización del espacio, auto segregación y participación ciudadana en la Ciudad de México, el caso de las calles cerradas de la zona de Coapa (Tlalpan, Distrito Federal). TRACE, procesos mexicanos y centroamericanos, (42), 71-78.

h t t p://dx.doi.org/10.22134/ trace.42.2002.536

Goytia Goyenechea, L. \& Martínez-Roldán, N. (2019). Conceptualización de la ciudad y su territorio. Tendencia geométrica del imaginario urbano. Estoa, 9(17), 79-92. https://doi.org/10.18537/est.v009.n017.a07

Harvey, D. (1992). Social justice, postmodernism and the city. International Journal of Urban and Regional Research, 16(4), 588-601. https://doi.org/10.1111/j.1468-2427.1992. tb00198.x

Harvey, D. (2008). The right to the city. Citado en Garnier, J.P. (2017). El derecho a la ciudad desde Henri Lefebvre hasta David Harvey. Entre teorizaciones y realización. Ciudades, 15(15), 217-225.

h t t p s://d o i.org/10.24197/ ciudades.15.2012.217-225

Hernández Vivero, G. (2017). Visualizador para el análisis de la justicia espacial como medida de accesibilidad a los servicios educativos del NMS en la Región X, Tejupilco, Estado de México [Tesis de licenciatura, UAEM].

http://hdl.handle.net/20.500.11799/71120

Hidalgo Páez, H. (2018). (In)justicia espacial en la ciudad de México [Tesis doctoral, UAM]. http://hdl.handle.net/11191/6439

Instituto Nacional de Estadística y Geografía (INEGI). (2020a). Encuesta Nacional de Seguridad Pública Urbana (ENSU) Tercer trimestre 2020

https://www.inegi.org.mx/app/saladeprensa/ noticia.html?id=5999

Instituto Nacional de Estadística y Geografía (INEGI). (2020b). Panorama nacional sobre la situación de la violencia contra las mujeres. http://library1.nida.ac.th/termpaper6/ sd/2554/19755.pdf
Iveson, K. (2011). Social or spatial justice? Marcuse and Soja on the right to the city. City, 15(2), 250-259.

https://doi.org/10.1080/13604813.2011.56 8723

Jasso López, L. C. (2015). ¿Por qué la Gente se siente insegura en el espacio público? (Tesis doctoral, CIDE).

https://cide.repositorioinstitucional.mx/ jspui/bitstream/1011/684/1/000145898 documento.pdf

Jian, I. Y., Luo, J. \& Chan, E. H. (2020). Spatial justice in public open space planning: Accessibility and inclusivity. Habitat International, 97, 1-10. https://doi.org/10.1016/j.habitatint.2020.102122

Lefebvre, H. (1972). La pensé marciste et la ville. Citado en Garnier, J.P. (2017). El derecho a la ciudad desde Henri Lefebvre hasta David Harvey. Entre teorizaciones y realización. Ciudades, 15(15), 217-225.

h t tps://doi . org/10.24197/ ciudades.15.2012.217-225

Ley General de Asentamientos Humanos, ordenamiento territorial y desarrollo urbano. (2016). Última reforma publicada DOF 1 de diciembre de 2020 (México).

http://www.diputados.gob.mx/LeyesBiblio/ pdf/LGAHOTDU_010621.pdf

Ley General de Desarrollo Social. (2004). Última reforma publicada DOF 25 de junio de 2018 (México).

http://www.ordenjuridico.gob.mx/ Documentos/Federal/pdf/wo83185.pdf

Marques Osorio, L. (2005). La carta mundial por el derecho a la ciudad. En N. Nehls Martínez \& M. L. Zárate (Eds.), El derecho a la ciudad en el mundo: compilación de documentos relevantes para el debate (pp. 163, 168). HIC-Latina.

ONU-Hábitat, por un mejor futuro. (2020, 24 de febrero). Componentes del derecho a la ciudad.

https://onuhabitat.org.mx/index.php/ componentes-del-derecho-a-la-ciudad

Orum, A. M., Steil, J. \& Connolly, J. (2019). Just City. En The Wiley Blackwell Encyclopedia of Urban and Regional Studies (pp. 1-6). https://doi.org/10.1002/9781118568446. eurs0169

Páramo, P. \& Burbano Arroyo, A. M. (2014). Los usos y la apropiación del espacio público para el fortalecimiento de la democracia. Revista de Arquitectura (Bogotá), 16(1), 6-15

https://doi.org/10.14718/RevArq.2014.16.2

Pérez, H. E. (2004). Percepción del espacio público. Bitácora Urbano Territorial, 1(8), 27-31.

https://revistas.unal.edu.co/index.php/ bitacora/article/view/18753

Pinzón Botero, M. V. \&y Echeverri Álvarez, I. C. (2010). Espacio público, cultura y calidad ambiental urbana una propuesta metodológica para su intervención. Investigación y Desarrollo, 18(1), 92-113.

http://www.redalyc.org/articulo. oa?id=26815364005

Pirie, G. H. (1983). On spatial justice. Environment \& Planning A: Economy and Space, 15(4), 465-473. https://doi.org/10.1068/a150465

Ramírez Kuri, P. (2015). Espacio público, ¿espacio de todos? Reflexiones desde la ciudad de México. Revista Mexicana de Sociología, 1(77), 7-36.

http://www.scielo.org.mx/pdf/rms/v77n1/ v77n1a1.pdf
Rawls, J. (1971) A theory of justice. Citado en Orum, A. M., Steil, J. \& Connolly, J. (2019). Just City. The Wiley Blackwell Encyclopedia of Urban and Regional Studies (pp. 1-6). https://doi.org/10.1002/9781118568446. eurs0169

Ribera Carbó, E. (2019). Plazas mayores y alamedas de México, una reflexión desde la geografía histórica. Investigaciones Geográficas, 100, 1-14.

https://doi.org/10.14350/rig.60017

Salamanca Villamizar, C., Barada, J. \& Beuf, A. (2019). (In)justicias espaciales y realidades latinoamericanas. Cuadernos de Geografía: Revista Colombiana de Geografía, 28(2), 209-224.

https://doi.org/10.15446/rcdg.v28n2.77327

Sánchez Almanza, A. (2018). Marco jurídico para la justicia socioespacial en la zona metropolitana del Valle de México. En A. Sánchez Almanza (Ed.), Calidad de vida en la zona metropolitana del Valle de México. Hacia la justicia socioespacial (pp. 45-66). Universidad Nacional Autónoma de México: Instituto de investigaciones Económicas.

http://ru.iiec.unam.mx/5032/1/Almanza\%20 libro\%20pdf.pdf

Sarmiento Pelayo, M. P. \& Fernández Moreno, A. (2019). Justicia social y diseño. Bitácora Urbano Territorial, 30(2), 11-24.

https://doi.org/10.15446/BITACORA. V30N2.81925

Segovia, O. \& Jordán, R. (2005). Espacios públicos urbanos, pobreza y construcción social. Revista Desarrollo y Sociedad, 30(122), 5-52.

https://repositorio.cepal.org/ handle/11362/5660

Silva, A. (2006). Imaginarios urbanos. 5ta. ed. Editorial Tercer Mundo.

https://imaginariosyrepresentaciones.files. wordpress.com/2015/05/silva-armandoimaginarios-urbanos.pdf

Soja, E. W. (2009). The city and spatial justice. Justice et Injustices Spatiales, 56-72. https://doi.org/10.4000/books.pupo.415

Soto Villagrán, P. (2012). El miedo de las mujeres a la violencia en la ciudad de México. Una cuestión de Justicia espacial. Revista Invi, 27, 145-169.

https://scielo.conicyt.cl/scielo. php?script $=$ sci_arttext $\&$ pid $=$ S0718$83582012000200005 \& \operatorname{lng}=$ en $\& n r m=i s o \&$ tlng $=$ en

Thiébaut, V. (7-8 de abril de 2011). Seminario: Cuestiones de género y justicia espacial desde la geografía [Resumen de seminario]. Cátedra Elisée Reclus, Centro de Estudios de Geografía Humana, Colegio de Michoacán.

http://www.scielo.org.mx/pdf/igeo/n75/ n75a15.pdf

Valera, S. (1999). Espacio privado, espacio público: Dialécticas urbanas y construcción de significados. Tres Al Cuarto, 6, 22-24. https://sistemamid.com/panel/uploads/ biblioteca/7097/7098/7110/7112/82817. pdf

Ziccardi, A. (2019). Las nuevas políticas urbanas y el derecho a la ciudad. En F. Carrión Mena \& M. Dammert Guardia (Eds.), Derecho a la ciudad, una evocación de las transformaciones urbanas en América Latina (pp. 61-95). Editorial CLACSO.

http://biblioteca.clacso.edu.ar/clacso/ gt/20200519104921/Derecho-a-la-ciudad. pdf \# page $=61$ 

(1) Portada: Las bovedadas de Bramante.

Fotografía: Luis Alberto Martínez Camacho (2021) CC BY-NC

\section{(ब) (1) (\$)}

(A) Orientación editorial

\section{Enfoque y alcance}

La Revista de Arquitectura (Bogotá) ( (ISSN 1657-0308 Impresa y E-ISSN 2357-626X en línea) es una publicación científica seriada de acceso abierto, arbitrada mediante revisión por pares (doble ciego) e indexada, en donde se publican resultados de investigación originales e inéditos.

Está dirigida a la comunidad académica y profesional de las áreas afines a la disciplina. Es editada por la Facultad de Diseño y el Centro de Investigaciones (CIFAR) de la Universidad Católica de Colombia en Bogotá (Colombia).

La principal área científica a la que se adscribe la Revista de Arquitectura (Bogotá) según la OCDE es:

Gran área: 6. Humanidades

Área: 6.D. Arte

Disciplina: 6D07. Arquitectura y Urbanismo

También se publican artículos de las disciplinas como 2A02, Ingeniería arquitectónica; 5C03, Estudios urbanos (planificación y desarrollo); 6D07, Diseño.

Los objetivos de la Revista de Arquitectura (Bogotá) son:

- Promover la divulgación y difusión del conocimiento generado a nivel local, nacional e internacional

- Conformar un espacio para la construcción de comunidades académicas y la discusión en torno a las secciones definidas.

- Fomentar la diversidad institucional y geográfica de los autores que participan en la publicación.

- Potenciar la discusión de experiencias e intercambios científicos entre investigadores y profesionales.

- Contribuir a la visión integral de la arquitectura, por medio de la concurrencia y articulación de las secciones mediante la publicación de artículos de calidad.

- Publicar artículos originales e inéditos que han pasado por revisión de pares, para asegurar que se cumplen las normas éticas, de calidad, validez científica, editorial e investigativa.

- Fomentar la divulgación de las investigaciones y actividades desarrolladas en la Universidad Católica de Colombia.
Palabras clave de la Revista de Arquitectura (Bogotá): arquitectura, diseño, educación arquitectónica, proyecto y construcción, urbanismo.

Idiomas de publicación: español, inglés, portugués y francés. Título abreviado: Rev. Arquit.

Titulo corto: RevArq

\section{Políticas de sección}

La revista se estructura en tres secciones correspondientes a las líneas de investigación activas y aprobadas por la institución, y dos complementarias, que presentan dinámicas propias de la Facultad de Diseño y las publicaciones relacionadas con la disciplina.

Cultura y espacio urbano. En esta sección se publican los artículos que se refieren a fenómenos sociales en relación con el espacio urbano, atendiendo aspectos de la historia, el patrimonio cultural y físico, y la estructura formal de las ciudades y el territorio.

Proyecto arquitectónico y urbano. En esta sección se presentan artículos sobre el concepto de proyecto, entendido como elemento que define y orienta las condiciones proyectuales que devienen en los hechos arquitectónicos o urbanos, y la forma como estos se convierten en un proceso de investigación y nuevo de conocimiento. También se presentan proyectos que sean resultados de investigación, los cuales se validan por medio de la ejecución y transformación en obra construida del proceso investigativo. También se contempla la publicación de investigaciones relacionadas con la pedagogía y didáctica de la arquitectura, el urbanismo y el diseño.

Tecnología, medioambiente y sostenibilidad. En esta sección se presentan artículos acerca de sistemas estructurales, materiales y procesos constructivos, medioambiente y gestión, relacionados con los entornos social-cultural, ecológico y económico.

Desde la Facultad. En esta sección se publican artículos generados en la Facultad de Diseño, relacionados con las actividades de docencia, extensión, formación en investigación o internacionalización, las cuales son reflejo de la dinámica y de las actividades realizadas por docentes, estudiantes y egresados; esta sección no puede superar el $20 \%$ del contenido.

Textos. En esta sección se publican reseñas, traducciones y memorias de eventos relacionados con las publicaciones en Arquitectura y Urbanismo.
A Frecuencia de publicación

Desde 1999 y hasta el 2015, la Revista de Arquitectura (Bogotá) publicó un volumen al año, a partir del 2016 se publicarán dos números por año en periodo anticipado, enero-junio y julio-diciembre, pero también maneja la publicación anticipada en línea de los artículos aceptados (versión Post-print del autor).

La Revista de Arquitectura (Bogotá) se divulga mediante versiones digitales (PDF, HTML, EPUB, XML) e impresascon un tiraje de 700 ejemplares, los tiempos de producción de estas versiones dependerán de los cronogramas establecidos por la editorial.

Los tiempos de recepción-revisión-aceptación pueden tardar entre seis y doce meses dependiendo del flujo editorial de cada sección y del proceso de revisión y edición adelantado.

Con el usuario y contraseña asignados, los autores pueden ingresar a la plataforma de gestión editorial y verificar el estado de revisión, edición o publicación del artículo.
A Canje

La Revista de Arquitectura (Bogotá) está interesada en establecer canje con publicaciones académicas, profesionales o científicas del área de Arquitectura y Urbanismo, como medio de reconocimiento y discusión de la producción científica en el campo de acción de la publicación.

Mecanismo

Para establecer canje por favor descargar, diligenciar y enviar el formato: RevArq FP20 Canjes

Universidad Católica de Colombia (2021,
junio-diciembre). Revista de
Arquitectura (Bogotá), 23(2),
I-132. Doi: 10.14718
ISSN: I657-0308
E-ISSN: 2357-626X
Especificaciones:
Formato: $34 \times 24 \mathrm{~cm}$
Papel: Mate II5 g
Tintas: Negro y policromía

A Contacto

Dirección postal:

Avenida Caracas N ${ }^{0} \cdot 46-72$ Universidad Católica de Colombia Bogotá D. C., Colombia Código postal: 111311

Facultad de Diseño Centro de Investigaciones (CIFAR) Sede El Claustro. Bloque "L", 4 piso Diag. 46A N ${ }^{0 .} 15 b-10$ Editor, Arq. César Eligio-Triana

Teléfonos:

+57 (1) $3277300-3277333$

Ext. $3109 ; 3112$ o 5146
Fax: +57 (1) 2858895

Correo electrónico:

revistadearquitectura@ucatolica.edu.co cifar@ucatolica.edu.co

Página WEB:

www.ucatolica.edu.co

Vínculo Revistas científicas

http://publicaciones.ucatolica.edu.co revistas-cientificas

https://revistadearquitectura.ucatolica.edu.co/ 
